DOI: $10.3724 /$ SP.J.1218.2013.00067

\title{
新型气动弯曲关节的静态特性分析
}

\author{
黄鹏程，杨庆华，鲍官军，王志恒，都明宇，张立彬 \\ （特种装备制造与先进加工技术教育部/浙江省重点实验室（浙江工业大学），浙江 杭州 310032）
}

\begin{abstract}
摘 要: 针对现有气动弯曲关节在低气压下弯曲角度受限制的问题, 将两个气动柔性驱动器 (FPA) 作为弯曲关 节驱动源, 利用结构夹角实现关节的弯曲动作, 并设计了一种新型气动弯曲关节. 该关节包括一个 $\mathrm{T}$ 型结构、两个 可以绕着转轴做圆周运动的转动结构和两个对称分布的 FPA, FPA 的两端固定在 $\mathrm{T}$ 型结构和转动结构上. 通过改变 两个 FPA 的内腔气压, 使 FPA 伸长推动转动结构转动, 使转动结构之间形成夹角, 从而实现整体结构的弯曲运动. 基于力矩平衡原理对弯曲关节中单个 FPA 的转动端进行静态特性分析. 仿真分析和实验表明, 相比于传统的单 FPA 弯曲关节, 新型弯曲关节的角度气压比更高, 即能在更低气压的条件下实现更大角度的弯曲任务. 同时, 其弯曲角 度和 FPA 的内腔气压呈非线性关系, 弯曲角度随关节结构参数的改变而改变.
\end{abstract}

关键词: 气动柔性驱动器 (FPA); 弯曲关节; 静态模型; 手指康复器

中图分类号: TP241

文献标识码: A

文章编号: 1002-0446(2013)-01-0067-06

\section{Static Characteristic Analysis of a New Type of Pneumatic Bending Joint}

\author{
HUANG Pengcheng, YANG Qinghua, BAO Guanjun, WANG Zhiheng, DU Mingyu, ZHANG Libin \\ (Key Laboratory of Special Purpose Equipment and Advanced Manufacturing Technology (Zhejiang University of Technology), \\ Ministry of Education \& Zhejiang Province, Hangzhou 310032, China)
}

\begin{abstract}
Aiming at the bending angle limit of existing pneumatic bending joints at low pressure, an idea of using structural angle to implement the bending action is proposed. And a new type of pneumatic bending joints with two flexible pneumatic actuators (FPAs) as its drive source is designed. This joint includes one T-type structure, two rotating structures which can perform circular motion around the hinge, and two symmetrical FPAs. And two symmetrical FPAs are fixed on the T-type structure and the rotating structure. The two FPAs stretch to push rotating structure to move when inner air pressures are changed, which leads to an angle between rotating structures, and thus bending motion of the joints is achieved. The static characteristics of the turning end of FPA on the bending joint are analyzed based on the moment equilibrium principle. Simulation analysis and experimental results show that angle-pressure ratio of the new pneumatic bending joint is higher than traditional ones. It means that the larger bending angle can be gotten at lower pressure. Besides, the bending angle of the bending joint is nonlinear with the air pressure inside the FPA and the bending angle changes with the structure parameters.
\end{abstract}

Keywords: flexible pneumatic actuator (FPA); bending joint; static model; finger rehabilitation apparatus

\section{1 引言( Introduction)}

气动柔性弯曲关节是气动柔性手部康复器结构 设计和控制的基础, 而此类弯曲关节目前多数采用 各种类型的气动柔性驱动器作为驱动源. 气动柔性 驱动器是一类以橡胶等柔顺性材料制作主要部件、 以压缩气体为动力的新型驱动装置, 其典型代表是 美国原子物理学家 McKibben 设计的 McKibben 驱 动器 ${ }^{[1-4]}$. 近年来, 随着气动柔性驱动器的深入研 究, 以其为基础设计的弯曲关节得到了进一步发展,
北华大学张金涛等人设计了双向主动弯曲气动柔性 关节 [5], 能较好地实现空间上的轴向伸长, 以及 2 维双向的可控弯曲; 浙江理工大学王龙辉等人提出, 用一对气动柔性驱动器并联相互对抗来驱动关节, 并以此设计了 7 自由度气动人工肌肉机械手臂 ${ }^{[6]}$.

浙江工业大学提出了一种在橡胶管内壁嵌入钢 丝来约束橡胶管轴向变形的气动柔性驱动器 $[7-8]$, 如图 1 所示, 并以此为基础设计了气动柔性弯曲关 节、扭转关节、侧摆关节等驱动关节 ${ }^{[9-12]}$, 其中弯曲 关节如图 2 所示. 这种弯曲关节柔顺性好、功率/质

基金项目: 国家 863 计划资助项目（2009AA04Z209）；国家自然科学基金资助项目（51075363）; 浙江省自然科学基金杰出青年团队项目（R1090674）; 中国博士后科学基金资助项目（2012M511385); 浙江省自然科学基金资助项目 (LY12E05022). 
量比大、动作平滑、噪声低、无污染, 但其弯曲性能 受到刚性结构参数、橡胶特性、橡胶管尺寸等因素 的影响, 同时其应用范围也受到限制, 尤其是在手 指康复器领域. 手指康复器对弯曲关节的尺寸有严 格的要求, 需要在小尺寸的前提下保证弯曲关节的 弯曲性能, 同时, 尽可能降低用于驱动的气体压力, 以免出现橡胶管与端盖连接处崩裂、漏气等意外情 况. 文 [13] 指出, 人类的手指关节有通用的弯曲角 度范围, 最大弯曲角度达到 $110^{\circ}$, 因此, 手指康复器 弯曲关节的设计必须保证 $110^{\circ}$ 之内的弯曲性能: 在 整体尺寸不超过人类手指关节大小的前提下，以低 气压驱动弯曲关节, 实现 $110^{\circ}$ 的弯曲任务.

本文基于 FPA 的基本特性, 提出一种适用于手 指康复器的新型气动弯曲关节, 详细描述了其结构 原理. 基于力矩平衡原理对单个 FPA 的转动端进行 静态特性分析, 并对其主要结构参数进行仿真分析, 通过实验验证建立的静态模型以及分析过程的准确 性.

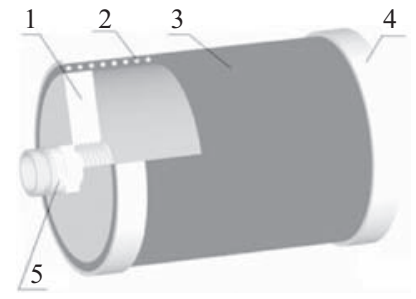

1 端盖; 2 螺旋弹簧; 3 橡胶管; 4 紧固圈; 5 管接头

图 1 气动柔性驱动器

Fig.1 Flexible pneumatic actuator

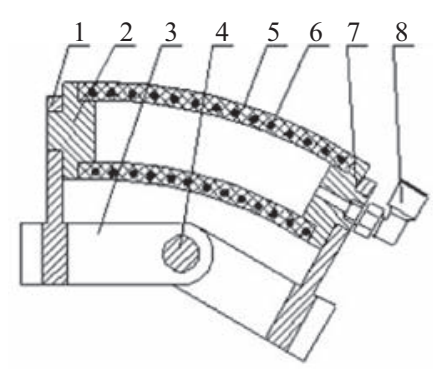

1 联接件; 2 左端盖; 3 连杆; 4 转轴; 5 橡胶管; 6 弹簧;

7 右端盖; 8 进气接头

图 2 弯曲关节

Fig.2 Bending joint

\section{2 结构原理（Structural principles）}

新型气动弯曲关节由一个 $\mathrm{T}$ 型结构、两个转动 结构和两个呈对称分布的 FPA 组成. FPA 的两端分 别固定在 $\mathrm{T}$ 型结构和转动结构上, $\mathrm{T}$ 型结构与转动 结构通过转轴连接, 转动结构可以相对于转轴做圆 周运动, 如图 3 所示. 当向两个 FPA 内腔充入压缩 气体时, FPA 伸长变形, 由于固定端的约束, FPA 只
能在转动端伸长变形, 从而推动转动结构围绕着转 轴转动一定的角度, 使左右两个转动结构形成夹角, 实现整体的弯曲动作; 当 FPA 内腔的气压降低时, 在橡胶弹性和弹簧的作用下, FPA 逐渐恢复原状, 拉动转动结构回到原位, 使弯曲关节恢复到初始状 态.

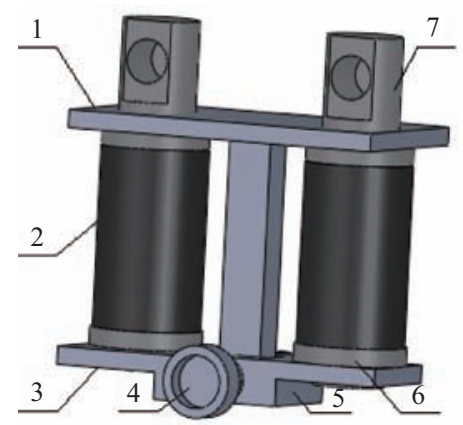

$1 \mathrm{~T}$ 型结构; $2 \mathrm{FPA} ; 3$ 转动结构; 4 转轴; 5 转动结构; 6 密封端端盖; 7 进气端端盖

图 3 双 FPA 弯曲关节

Fig.3 Double FPA bending joint

\section{3 静态模型（Static model）}

新型气动弯曲关节的运动过程如下: 通过向 FPA 内腔充气, 使 FPA 伸长变形, 从而推动下端转 动结构围绕转轴做圆周运动. 分析建立弯曲关节数 学模型之前, 做如下假设:

(1) 构成弯曲关节的两个 FPA 完全一样;

(2) FPA 在变形过程中平均半径保持不变;

(3) FPA 在伸长变形, 推动转动结构做圆周运 动的过程中, 其对转动结构的作用力垂直于转动结 构.

\section{1 弯曲关节 FPA 壁厚分析}

在压力为 $P$ 的压缩气体作用下, 转动结构转过 一定的角度 $\alpha$, 两个转动结构夹角为 $\theta$. 此时弯曲关 节的弯曲角度为 $2 \alpha$. 由于弯曲关节的两个 FPA 呈 左右对称分布, 因此对 FPA 进行变形分析, 如图 4 所示.

图 4 中, 图中 1-1 为 FPA 橡胶管的横截面, $B C$ 为 $\mathrm{FPA}$ 的固定端, $E F$ 是 $\mathrm{FPA}$ 的转动端, 点 $E$ 是 转轴在平面上的投影点, 而转动结构的转动角度 $\angle C O F=\alpha$, 平面 $B C F E$ 为通过 FPA 轴心线的横断 面, 而平面 $B^{\prime} C^{\prime} F^{\prime} E^{\prime}$ 为平行于横断面的平面. 由于 FPA 的两个端面分别固定在 $\mathrm{T}$ 型结构和转动结构 上, 则 $B C$ 和 $E F$ 长度固定, 且 $B C=E F=H$. 同 时, $B E$ 的长度固定等于弯曲关节 $\mathrm{FPA}$ 的初始长度 $L_{0}$. 定义 FPA 端面上 $\angle C O^{\prime \prime} C^{\prime}=\phi, 0 \leqslant \phi \leqslant 2 \pi$, 则弧 $C^{\prime} F^{\prime}$ 的长度随角度 $\phi$ 的变化而变化, 定义 $C^{\prime} F^{\prime}$ 的弧 
长为 $L_{\phi}$.

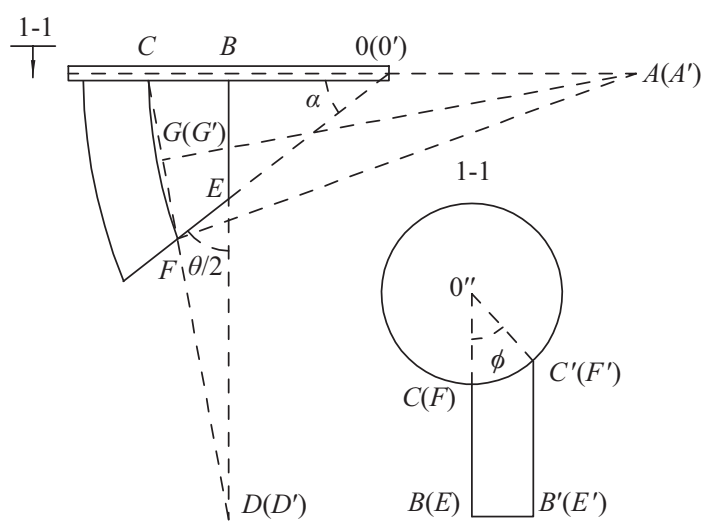

图 4 FPA 变形分析

Fig.4 Deformation analysis on FPA

在 $\triangle C O F$ 中:

$$
\begin{aligned}
& O C=O B+B C=\frac{L}{\tan \alpha}+H \\
& O F=O E+E F=\frac{L}{\sin \alpha}+H
\end{aligned}
$$

考虑 $\phi$ 的影响, 同时令 $O^{\prime} C^{\prime}=a, O^{\prime} F^{\prime}=b$, 可 得:

$$
\begin{aligned}
& a=\frac{L}{\tan \alpha}+H+r_{0}(1-\cos \phi) \\
& b=\frac{L}{\sin \alpha}+H+r_{0}(1-\cos \phi)
\end{aligned}
$$

则:

$$
L_{\mathrm{s}}=C^{\prime} F^{\prime}=\sqrt{a^{2}+b^{2}-2 a b \cos \alpha}
$$

式中 $r_{0}$ 是弯曲关节 FPA 的平均半径.

在直角 $\triangle B^{\prime} C^{\prime} D$ 和 $\triangle C^{\prime} O^{\prime} F$ 中:

$$
\begin{aligned}
\cos \angle O^{\prime} C^{\prime} F^{\prime} & =\cos \left(90^{\circ}-\angle B^{\prime} D^{\prime} C^{\prime}\right) \\
& =\sin \angle B^{\prime} D^{\prime} C^{\prime} \\
& =\left(C^{\prime} O^{\prime 2}+C^{\prime} F^{\prime 2}-O^{\prime} F^{\prime 2}\right) /\left(2 C^{\prime} O^{\prime} \cdot C^{\prime} F^{\prime}\right) \\
& =\left(L_{\mathrm{s}}^{2}+a^{2}-b^{2}\right) /\left(2 a \cdot L_{\mathrm{s}}\right) \\
& =(a-b \cos \alpha) / L_{\mathrm{s}}
\end{aligned}
$$

在直角 $\triangle B^{\prime} C^{\prime} D^{\prime}$ 和直角 $\triangle G^{\prime} C^{\prime} A^{\prime}$ 中:

$$
\begin{aligned}
& \sin \angle G^{\prime} A^{\prime} C^{\prime}=\sin \angle B^{\prime} D^{\prime} C^{\prime}=\frac{a-b \cos \alpha}{L_{\mathrm{s}}} \\
& A^{\prime} C^{\prime}=\frac{C^{\prime} G^{\prime}}{\sin \angle G^{\prime} A^{\prime} C^{\prime}}=\frac{L_{\mathrm{s}}^{2}}{2(a-b \cos \alpha)}
\end{aligned}
$$

那么, 在扇形 $A^{\prime} C^{\prime} E^{\prime}$ 中可求得 $C^{\prime} F^{\prime}$ 弧长

$$
\begin{aligned}
L_{\phi} & =A^{\prime} C^{\prime} \cdot 2 \angle C^{\prime} A^{\prime} G^{\prime} \\
& =\frac{L_{\mathrm{s}}^{2}}{a-b \cos \alpha} \arcsin \frac{a-b \cos \alpha}{L_{\mathrm{s}}}
\end{aligned}
$$

由壁厚公式 $t_{\phi}=t_{0} \cdot L_{0} / L_{\phi}$, 可得到在 $\phi$ 处的橡 胶管壁厚 $t_{\phi}$ 为

$$
t_{\phi}=\frac{t_{0} L_{0}(a-b \cos \alpha)}{\left(a^{2}+b^{2}-2 a b \cos \alpha\right) \arcsin \frac{a-b \cos \alpha}{L_{\mathrm{s}}}}
$$

式中 $L_{0}$ 和 $t_{0}$ 分别为弯曲关节 FPA 的初始长度和初 始壁厚.

\section{2 弯曲关节静态模型}

对弯曲关节单个 FPA 的自由端 (即转板) 进行 力矩平衡分析, 如图 5 所示. $M_{\mathrm{a}}$ 为橡胶管的弹性力 产生的力矩, $M_{\mathrm{p}}$ 为关节 FPA 内腔压力 $P$ 与大气压 力 $P_{\mathrm{atm}}$ 在端面产生的力矩. 注: 图中 1-1 为 FPA 橡 胶管的横截面.

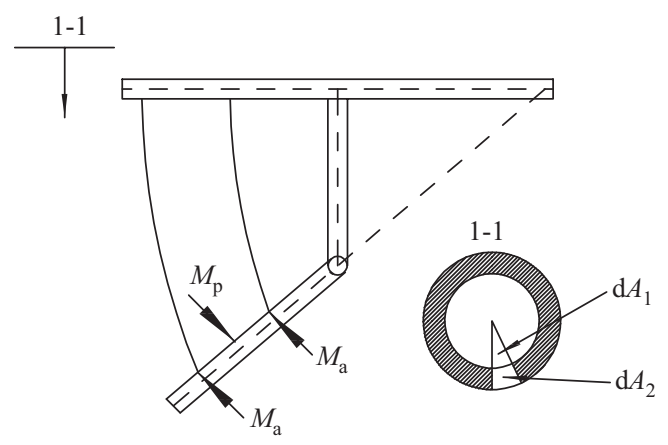

图 5 弯曲关节 FPA 力矩平衡分析

Fig.5 Equilibrium of moment for FPA on bending joint

在外力矩为 0 的情况下, 力矩平衡方程: $M_{\mathrm{a}}=$ $M_{\mathrm{p}}$. 其中:

$$
\begin{aligned}
\mathrm{d} M_{\mathrm{p}} & =L_{\mathrm{p}} \cdot \mathrm{d} F_{\mathrm{p}} \\
& =L_{\mathrm{p}}\left(P-P_{\mathrm{atm}}\right) \mathrm{d} A_{1} \\
& =L_{\mathrm{p}}\left(P-P_{\mathrm{atm}}\right) r \mathrm{~d} r \mathrm{~d} \phi \\
\mathrm{d} M_{\mathrm{a}} & =L_{\mathrm{a}} \cdot \mathrm{d} F_{\mathrm{a}}
\end{aligned}
$$

式中 $L_{\mathrm{p}}$ 是力 $\mathrm{d} F_{\mathrm{p}}$ 相对于 $E$ 的力臂长度, $L_{\mathrm{a}}$ 是力 $\mathrm{d} F_{\mathrm{a}}$ 相对于 $E$ 的力臂长度.

由图 5 可知

$$
L_{\mathrm{P}}=L_{\mathrm{a}}=H+r_{0} \cdot(1-\cos \phi)
$$

因此:

$$
\begin{aligned}
M_{\mathrm{p}} & =2 \int_{0}^{\pi} \int_{0}^{r_{0}} L_{\mathrm{p}}\left(P-P_{\mathrm{atm}}\right) r \mathrm{~d} r \mathrm{~d} \phi \\
& =\left(P-P_{\mathrm{atm}}\right) r_{0}^{2} \pi\left(H+r_{0}\right)
\end{aligned}
$$

同时，

$$
\begin{aligned}
\mathrm{d} F_{\mathrm{a}} & =\sigma \cdot \mathrm{d} A_{2}=E \cdot \frac{L_{\phi}-L_{0}}{L_{0}} \cdot \mathrm{d} A_{2} \\
& =E \cdot \frac{L_{\phi}-L_{0}}{L_{0}} \cdot t_{\phi} \cdot r_{0} \mathrm{~d} \phi
\end{aligned}
$$


式中 $\sigma$ 和 $E$ 分别为弯曲关节 FPA 橡胶管应力和弹 性模量.

$$
\begin{aligned}
M_{\mathrm{a}} & =\int L_{\mathrm{a}} \cdot \mathrm{d} F_{\mathrm{a}}^{\prime} \\
& =2 \int_{0}^{\pi}\left[H+r_{0} \cdot(1-\cos \phi)\right] E \frac{L_{\phi}-L_{0}}{L_{0}} t_{\phi} r_{0} \mathrm{~d} \phi \\
& =2 \pi E r_{0} t_{0}\left(H+r_{0}\right)-2 E r_{0} t_{0} L_{0} \int_{0}^{\pi} \frac{H+r_{0}(1-\cos \phi)}{L_{\phi}} \mathrm{d} \phi
\end{aligned}
$$

由 $M_{\mathrm{a}}=M_{\mathrm{p}}$ 得:

$$
\begin{aligned}
\Delta P=P-P_{\mathrm{atm}}= & \frac{2 E t_{0}}{r_{0}}-\frac{2 E t_{0} L_{0}}{\pi r_{0}\left(H+r_{0}\right)} . \\
& \int_{0}^{\pi} \frac{\left[H+r_{0}(1-\cos \phi)\right](a-b \cos \alpha)}{L_{\mathrm{s}}^{2} \arcsin \left[(a-b \cos \alpha) / L_{\mathrm{s}}\right]} \mathrm{d} \phi
\end{aligned}
$$

其中:

$$
\begin{aligned}
& a=\frac{L}{\tan \alpha}+H+r_{0}(1-\cos \phi) \\
& b=\frac{L}{\sin \alpha}+H+r_{0}(1-\cos \phi) \\
& L_{\mathrm{s}}=\sqrt{a^{2}+b^{2}-2 a b \cos \alpha}
\end{aligned}
$$

\section{4 仿真分析 (Simulation analysis)}

由式 (13) 可得, 弯曲关节弯曲角度 $\theta$ 与其 FPA 的内腔气压 $P 、 \mathrm{FPA}$ 初始壁厚 $t_{0} 、 \mathrm{FPA}$ 初始长度 $L_{0}$ 、 $\mathrm{FPA}$ 平均半径 $r_{0} 、 \mathrm{FPA}$ 橡胶管橡胶弹性模量 $E$ 和 FPA 位置参数 $H$ 有关. 根据式 (13) 和表 1 所示参 数, 对弯曲关节结构参数与其弯曲角度的关系进行 仿真分析.

表 1 弯曲关节 FPA 静态模型仿真参数

Tab.1 Simulation parameters of bending joint FPA static model

\begin{tabular}{c|c}
\hline 参数 & 数值 \\
\hline 初始壁厚 $t_{0} / \mathrm{mm}$ & 2.0 \\
\hline 初始长度 $L_{0} / \mathrm{mm}$ & 20.0 \\
\hline 平均半径 $r_{0} / \mathrm{mm}$ & 5.0 \\
\hline FPA 位置参数 $H / \mathrm{mm}$ & 5.0 \\
\hline 弹性模量 $E / \mathrm{MPa}$ & 2.0 \\
\hline 大气压力 $P_{\mathrm{atm}} / \mathrm{MPa}$ & 0.1 \\
\hline
\end{tabular}

图 6 是新型弯曲关节与相同尺寸的传统弯曲关 节的弯曲性能理论曲线对比图. 从图中可知, 当弯 曲角度相同时, 新型弯曲关节所需的内腔气压小于 传统弯曲关节, 即前者的弯曲角度气压比远大于后 者. 因此, 新型弯曲关节的弯曲性能好于传统的弯 曲关节。

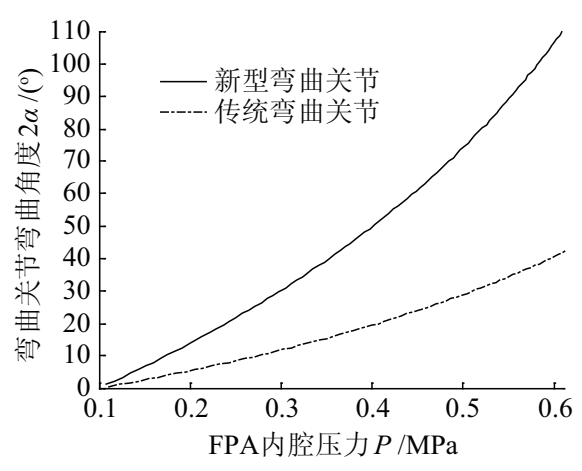

图 6 弯曲性能对比图

Fig.6 Bending performance comparison

图 $7 \sim 10$ 分别是 $2 \alpha=110^{\circ}$ 时, FPA 内腔气压 和弯曲关节各参数之间的关系曲线. 由图 7 可知, FPA 内腔所需气压 $P$ 随着 $L$ 增大而减小; 由图 8 所 示的曲线关系可知, FPA 内腔所需气压 $P$ 随着 $r$ 增 大而减小; 图 9 的关系曲线指出, FPA 内腔所需气压 $P$ 随着 $t$ 增大而增大; 由图 10 的关系曲线可见, FPA 内腔所需气压 $P$ 随着 $H$ 增大而增大.

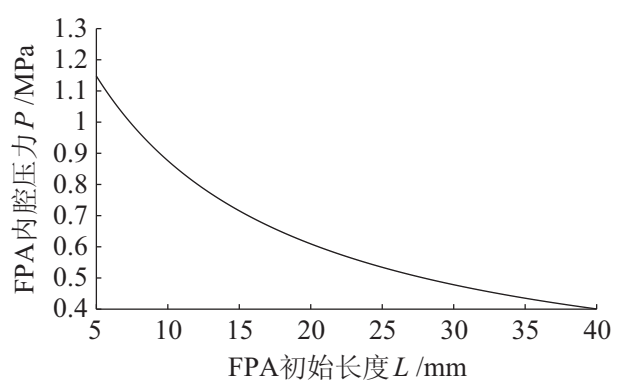

图 $7 L$ 与 $P$ 的关系

Fig.7 Relationship between $L$ and $P$

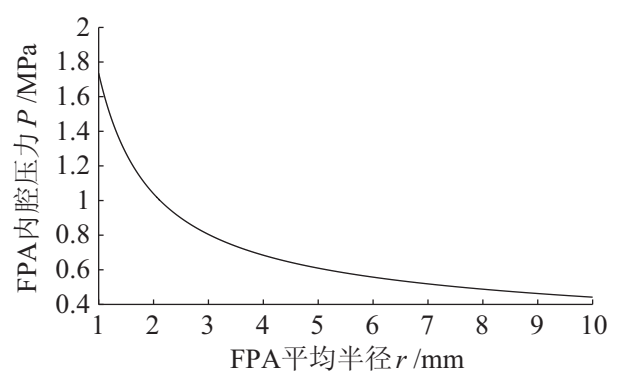

图 $8 r$ 与 $P$ 的关系

Fig.8 Relationship between $r$ and $P$

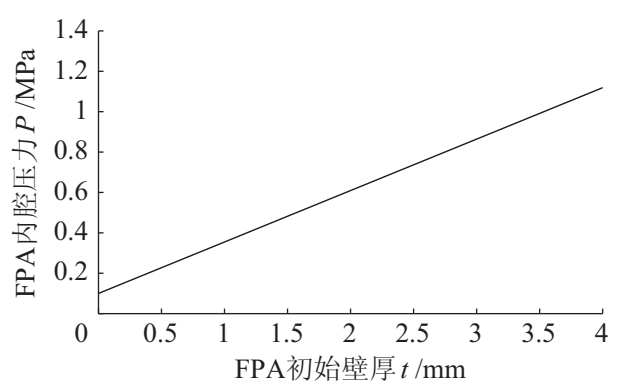

图 $9 t$ 与 $P$ 的关系

Fig.9 Relationship between $t$ and $P$ 


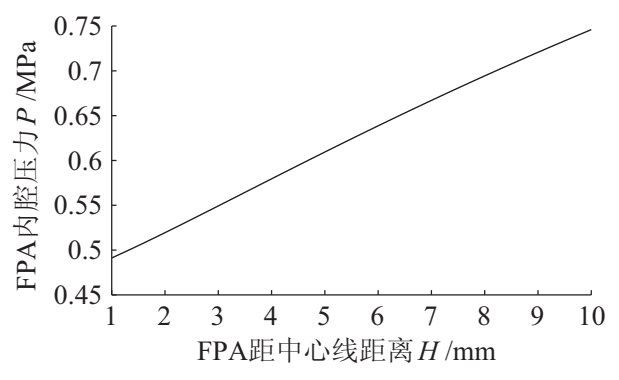

图 $10 H$ 与 $P$ 的关系

Fig.10 Relationship between $H$ and $P$

本文根据课题组成员左手食指平均尺寸, 按照 仿真分析的结果, 设计如表 1 所示参数的弯曲关节, 以用于康复实验. 在实际应用中, 可以根据患者具 体情况, 通过调节关节的结构参数获得所需的角度 气压比, 从而达到结构的最优化设计.

\section{5 实验结果与分析 (Experiment results and analysis )}

实验原理如图 11 所示. 实验中用到的弯曲关节 参数如表 1 所示. 向弯曲关节的两个 FPA 内腔充入 压缩气体, 测量关节的弯曲角度. 为了消除橡胶的 粘滞性带来的影响, 每次对弯曲关节充入压缩气体 之后停留 $3 \mathrm{~s} \sim 5 \mathrm{~s}$, 然后再记录下弯曲关节的角度 数据. 同时, 为了尽量减小客观原因对实验结果造 成的影响, 对整个弯曲过程进行 5 次重复实验, 取 相应气压的弯曲角度平均值作为最后的实验结果.

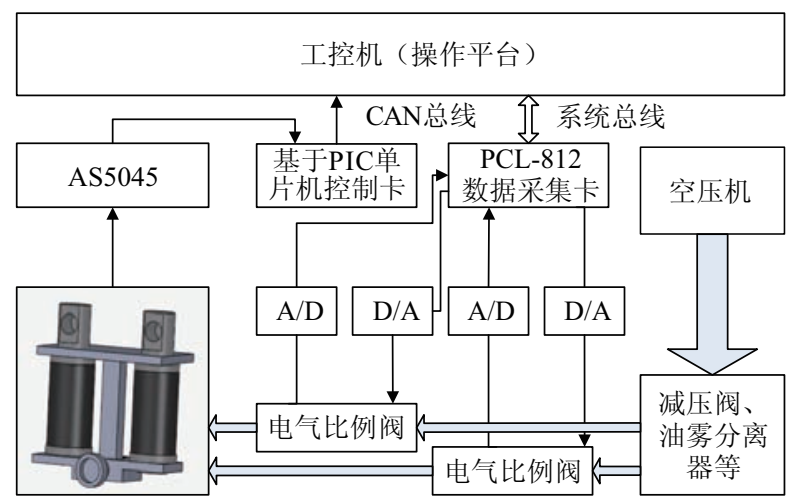

图 11 弯曲关节原理图

Fig.11 Schematic diagram of the bending joint

实验结果与仿真曲线如图 12 所示. 弯曲关节实 物如图 13 所示. 由实验结果和仿真结果的对比可 知: 当 FPA 内腔气压在 $0.1 \mathrm{MPa} \sim 0.35 \mathrm{MPa}$ 之间时, 实际弯曲角度与理论值偏差较小, 曲线吻合较好; 随 着 FPA 内腔气压增大, 偏差也随之增大, 实际弯曲 角度低于理论值; 当FPA 内腔气压达到 $0.61 \mathrm{MPa}$ 时, 理论弯曲角度达到最大期望角度 $110^{\circ}$, 此时实际弯 曲角度值为 $102.5^{\circ}$, 偏差值为 $7.5^{\circ}$, 偏差率为 $6.8 \%$.
总体来看, 实验数据与仿真曲线基本吻合, 建立的 数学模型能基本反映新型弯曲关节的静态特性.

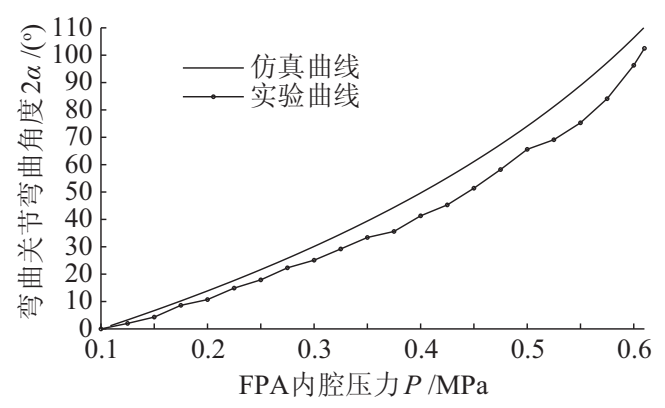

图 12 弯曲关节静态实验仿真曲线

Fig.12 Simulation curve of bending joint static experiment

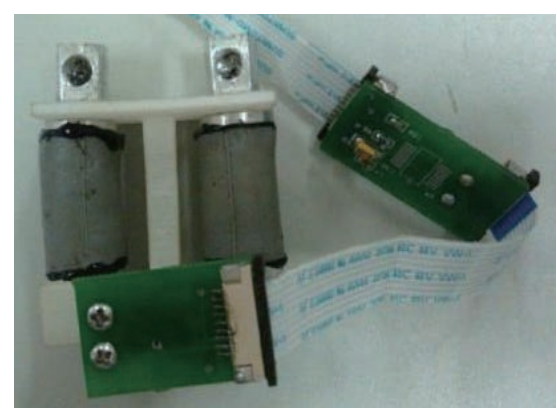

图 13 双 FPA 气动柔性弯曲关节

Fig.13 Flexible pneumatic bending joint with double FPAs

分析其产生偏差的原因, 有以下几点:

(1) FPA 的制作过程和材料等因素导致的弯曲 关节左右 FPA 性能有所差异;

(2) 充气过程中, 气体回路漏气等因素引起的 FPA 内腔气压不一致;

(3) 电气比例阀在向弯曲关节输出气压时, 产生 关节抖动引起的偏差;

(4) 测量角度并反馈过程中产生的偏差.

\section{6 结论 (Conclusions)}

提出一种以双 FPA 作为驱动源, 利用结构夹角 实现弯曲动作的新型气动柔性弯曲关节. 相比于传 统弯曲关节, 该弯曲关节弯曲性能有较大提高, 能 满足手指康复器的研究应用需求.

针对单个 FPA 进行力矩平衡分析, 并以此建立 其静态模型. 仿真与实验结果表明, 建立的数学模 型较为准确, 能反映弯曲关节的静态特性.

\section{参考文献（References）}

[1] Caldwell D G, Medrano-Cerda G A, Goodwin M J. Braided pneumatic actuator control of a multi-jointed manipulator[C]// IEEE International Conference on Systems, Man and Cybernetics. Piscataway, NJ, USA: IEEE, 1993: 423-428.

[2] Chou C P, Hannaford B. Static and dynamic characteristics of McKibben pneumatic artificial muscles[C]//IEEE International 
Conference on Robotics and Automation. Piscataway, NJ, USA: IEEE, 1994: 281-286.

[3] Chou C P, Hannaford B. Measurement and modeling of McKibben pneumatic artificial muscles[J]. IEEE Transactions on Robotics and Automation, 1996, 12(1): 90-103.

[4] Tsagarakis N, Caldwell D G. Improved modelling and assessment of pneumatic muscle actuators[C]//IEEE International Conference on Robotics and Automation. Piscataway, NJ, USA: IEEE, 2000: 3641-3646.

[5] 张金涛, 耿德旭, 刘晓敏, 等. 双向主动弯曲气动柔性关节 力学特性分析 [J]. 液压与气动, 2011(6): 39-42.

Zhang J T, Geng D X, Liu X M, et al. Mechancal characteristics analysis of two-way active bending flexible pneumatic joint[J]. Chinese Hydraulics \& Pneumatics, 2011(6): 39-42.

[6] 王龙辉, 金英子, 朱红亮, 等. 七自由度气动人工肌肉机械 手臂的设计及研究 [J]. 浙江理工大学学报, 2012, 29(1): 7478.

Wang L H, Jin Y Z, Zhu H L, et al. Design and research of seven degrees of freedom robotic arm driven by pneumatic artificial muscle[J]. Journal of Zhejiang Sci-Tech University, 2012, 29(1): 74-78.

[7] 张立涁, 鲍官军, 杨庆华, 等. 气动柔性扭转关节静态模型 [J]. 机械工程学报, 2008, 44(7): 134-138.

Zhang L B, Bao G J, Yang Q H, et al. Static model of flexible pneumatic torsion joint[J]. Journal of Mechanical Engineering, 2008, 44 (7): 134-138

[8] 杨庆华, 张立彬, 胥芳, 等. 气动柔性弯曲关节的特性及其 神经 PID 控制算法研究 [J]. 农业工程学报, 2004, 20(4): 8891.

Yang Q H, Zhang L B, Xu F, et al. Investigation of the characteristics of pneumatic flexible-bending joint and its neural PID controlling algorithm[J]. Transactions of the Chinese Society of
Agricultural Engineering, 2004, 20(4): 88-91.

[9] 钱少明, 杨庆华, 鲍官军, 等. 基于气动柔性驱动器的弯曲 关节的基本特性研究 [J]. 中国机械工程，2009(24)：29032907.

Qian S M, Yang Q H, Bao G J, et al. Research on basic characteristics of bending joint based on flexible pneumatic actuator[J]. China Mechanical Engineering, 2009(24): 2903-2907.

[10] 鲍官军, 王志恒, 杨庆华, 等. 气动柔性扭转关节动态特性 研究 [J]. 农业机械学报, 2010, 41(6): 204-207,226.

Bao G J, Wang Z H, Yang Q H, et al. Dynamic characteristics of flexible pneumatic torsion joint[J]. Transactions of the Chinese Society of Agricultural Machinary, 2010, 41(6): 204-207,226.

[11] 鲍官军, 邵铁峰, 李尚会, 等. 气动柔性摆动关节静态模型 [J]. 农业机械学报, 2011，42(6): 198-202,212.

Bao G J, Shao T F, Li S H, et al. Static model of flexible pneumatic swaying joint $[\mathrm{J}]$. Transactions of the Chinese Society of Agricultural Machinary, 2011, 42(6): 198-202,212.

[12] 王志恒, 杨庆华, 钱少明, 等. 气动侧摆关节的动态特性 [J]. 农业工程学报, 2012，28(7): 19-26。

Wang Z H, Yang Q H, Qian S M, et al. Dynamic characteristics of pneumatic side-sway joint[J]. Transactions of the Chinese Society of Agricultural Engineering, 2012, 28(7): 19-26.

[13] 张付祥. 创伤手指康复机械手系统研究 [D]. 哈尔滨: 哈尔 滨工业大学, 2007.

Zhang F X. Research on the system of the rehabilitation manipulator for injured fingers[D]. Harbin: Harbin Institute of Technology, 2007.

\section{作者简介:}

黄鹏程 (1986-), 男, 博士生. 研究领域: 手指运动仿生康 复器. 